\title{
ENTRE MÉXICO \\ Y LA IV INTERNACIONAL: \\ EL PRT Y LA LIBERACIÓN DE LAS MUJERES
}

\section{BETWEEN MEXICO \\ AND THE FOURTH INTERNATIONAL:}

THE PRT AND WOMEN'S LIBERATION

\author{
Ana Sofía Rodríguez Everaert \\ (iD) orcid.org/0000-0002-8079-9195 \\ El Colegio de México \\ México \\ asrodriguez@colmex.mx
}

\begin{abstract}
This article analyzes the agenda with respect to women's liberation during the first years of the Partido Revolucionario de los Trabajadores, Mexican section of the Fourth International. Based on the testimonies of militants, programmatic documents and publications of the PRT, as well as communiqués from feminist groups in Mexico and the central IV International, it explains, first of all, the sources and forms that these ideas took in the political group, and some of its programmatic and political consequences that differenciate this organization from other leftist political parties in Mexico.
\end{abstract}

Keywords: trotskyism, second-wave feminism, political parties, Third-world.

\section{Resumen}

Este artículo analiza la agenda de los primeros años del Partido Revolucionario de los Trabajadores (PRT), sección mexicana de la IV Internacional, con respecto a la liberación de la mujer. A partir de testimonios de militantes, documentos programáticos y publicaciones del PRT, así como de comunicados de agrupaciones feministas en México y de la IV Internacional central, se explican, en primer lugar, las formas que cobró este ideario en la agrupación política, sus fuentes, y algunos de sus alcances programáticos y políticos que distinguen a este organismo de otros partidos políticos de izquierda en México.

Palabras clave: trotskismo, feminismo de la segunda ola, partidos políticos, Tercer mundo. 


\section{Introducción}

Amalia García, exmilitante del Partido Comunista Mexicano (PCM), recuerda que a finales de la década de 1970 ella y sus compañeras colgaron una pancarta afuera del Auditorio Nacional de la Ciudad de México en la que se leía: "Proletarios del mundo... ¿quién lava sus calcetines?” (García, 2020). La intervención tuvo lugar durante una emisión del Festival "Oposición" en la que las comunistas mexicanas retomaban una de las consignas surgidas del mayo francés que, parafraseando irónicamente la última frase del Manifiesto del Partido Comunista, cuestionaba a sus compañeros de partido desde la perspectiva del feminismo (Comer, 2018).

Era una más de las manifestaciones del feminismo de la segunda ola que se abrió paso en México durante esa década, pero que demostraba un dilema concreto: el de las tensiones entre el feminismo y las izquierdas marxistas. Presentes desde finales del siglo XIX, estas tiesuras derivaban del presunto dilema estratégico sobre priorizar la lucha de clases a cualquier otra reivindicación, incluida la discriminación hacia las mujeres, dada su dimensión social primigenia (Honeycut, 1976).

Siglo y medio más tarde, muchas corrientes marxistas no habían logrado darle la vuelta a este dilema, según prueba la anécdota de García. Pero éste no fue el caso del trotskismo mexicano, que se había esforzado por atender las demandas de sus militantes mujeres, aunque con alcances diferenciados. La sección nacional de la IV Internacional, el Partido Revolucionario de los Trabajadores (PRT), se manifestó por la defensa de las mujeres muy poco tiempo después de formarse.

En los programas y publicaciones de este partido, pero sobre todo entre sus militantes, el feminismo cobró suficiente importancia como para que en 1977, a un año de su fundación, se creara una Comisión de la Mujer que existiría hasta 1992, año en que el partido vivió una de sus escisiones más fuertes (Oranday Dávila, 2015: 194; Monk, 2020).
Recientemente, las mujeres que se identificaron y trabajaron con el feminismo dentro del PRT han decidido contar su historia (López Rosado y Márquez Gileta, 2019). ' Los recuerdos de estas militantes dan cuenta de las lecturas, espacios y causas que confirman la existencia de una rica discusión teórica -además de trabajo de base-sobre los ejes del feminismo y la lucha de clases. En este texto se estudia la manera en que el tema de la liberación de la mujer se construyó en el PRT, sus fuentes y algunos de sus resultados en los primeros años de vida de la organización, a partir de los recuerdos de estas mujeres y el análisis de documentos del partido. Además de recurrir a los testimonios reunidos por López Rosado y Márquez Gileta, esta investigación recoge la información de dos entrevistas a profundidad con exmilitantes del PRT, el análisis de artículos sobre el tema de la mujer en las publicaciones periódicas del partido y otros documentos programáticos de la organización. Asimismo, fueron consideradas fuentes sobre las discusiones en la directiva de la IV Internacional en esos años, lo mismo que del feminismo no partidista en México, con la intención de poder situar y entender las particularidades del ideario de la liberación de la mujer impulsada dentro del partido trotskista mexicano.

Dado que la intención es analizar cómo se incorporó el feminismo al trotskismo mexicano, este estudio se restringe a la década de los setenta del siglo pasado, inmediatamente antes de la fundación del PRT y durante los primeros años del partido en funciones. Aunque el feminismo se mantuvo como un eje importante durante toda la existencia del PRT, para analizar a cabalidad los cambios y permanencias del tema de la mujer en la vida del partido frente a coyun-

\footnotetext{
1 El libro de María Stella Oranday aquí citado es un esfuerzo anterior por narrar la experiencia del PRT con la causa de las mujeres, también en boca de una de sus militantes. Este libro, si bien no profundiza en el análisis del origen del feminismo en el partido ni en sus fundamentos teóricos, es un repaso sistemático de los eventos que avanzaron la discusión sobre los derechos de las mujeres dentro del PRT, nutrido de una enorme cantidad de fuentes documentales que la autora conserva en su archivo personal.
} 
turas muy complejas -las elecciones de 1982 y 1988, una fuerte escisión en 1985 y la posterior ruptura de los trotskistas en el contexto del zapatismo- se requerirían otras fuentes y aproximaciones. Sin embargo, el periodo aquí estudiado y las particularidades ideológicas del PRT que resultaron de la incorporación del feminismo y otras demandas basadas en el lenguaje de los derechos, nos dan pistas para entender el devenir de la organización en el panorama de la izquierda nacional.

Este texto busca mostrar y explicar la relación entre el trotskismo mexicano y el feminismo, hasta ahora estudiada someramente en algunos recuentos históricos del feminismo de la segunda ola en México (Bartra, 1999; Lau Jaiven, 2006). Al mismo tiempo, estudia una dimensión de la sección de la IV Internacional en México ignorada en los estudios del trotskismo en México (Rodríguez Araujo, 2015) y abre vetas de comparación con otras secciones del trotskismo internacional que podrían nutrir la historia de la IV Internacional en el periodo que va de 1969 a 1990 y que concierne especialmente a América Latina. Finalmente, éste también es un trabajo que permite pensar en algunas formas de subjetivación y socialización dentro de los partidos y la militancia en función del género, con lo cual se suma a una historiografía incipiente pero promisoria (Hughes, 2012). Éste es un tema que está por estudiarse a profundidad en México y que permitiría evaluar desde otros ángulos el funcionamiento de estas organizaciones partidistas y sus idearios, en el contexto de la reforma política y el pluralismo político de las últimas décadas del siglo XX mexicano.

\section{La construcción de un feminismo trotskista}

Tras casi una década de llamados a la unidad de las fuerzas de izquierda por parte del Grupo Comunista Internacionalista -de alineación trotskista-, en 1976 se fundó en México el Partido Revolucionario de los Trabajadores (PRT) $(\mathrm{GCl}, 1971){ }^{2}$ Éste buscaba ser un

2 Reunía al Grupo Comunista Internacionalista (des- partido capaz de "dirigir a la clase obrera", independiente de "estalinistas y burócratas" (Estatutos del PRT, 1976, en Oranday Dávila, 2015: 335). Un año después de su fundación, el PRT organizó un Congreso extraordinario para evaluar el trabajo realizado hasta entonces y aquello que habría de impulsarse en el futuro próximo, considerando la crisis económica con la que se clausuraba el gobierno de Luis Echeverría. Los ejes de trabajo que resultaron de las discusiones en el congreso consideraban la democracia sindical, la Ley Federal Electoral, la libertad de expresión y de manifestación, la liberación de los presos políticos, la reforma urbana, la protección del campo y las minorías étnicas, así como la liberación de las mujeres (PRT, 1977a). Este último punto, extraño entre los partidos del tipo, condensaba el activismo feminista de las mujeres provenientes de las distintas facciones trotskistas que existían antes de su unificación en el PRT. En sus distintos grupos, las militantes se habían empapado de las demandas por la liberación de las mujeres que sus camaradas en otras partes del mundo llevaban articulando, desde la década de 1960, en textos y reuniones. En esos primeros años de vida del PRT, la lucha por las mujeres integraba esta agenda compartida, pero también incluía lecturas teóricas popularizadas entre los militantes mexicanos mediante formas y mecanismos propios de su contexto inmediato. Y más importante, una forma de comunicarlas que abrevaba lo mismo de las directrices de la IV Internacional que de la organización de este partido local.

Los intercambios que permitía la red de la IV Internacional proveían apoyo, legitimidad y estrategia. Cuenta Heather Dashner Monk, quien se incorporó a las filas del trotskismo mexicano después de llegar a México desde Estados Unidos en 1974, que las oficinas centrales de la Internacional se había propuesto aprobar un resolutivo en relación al trabajo con las mujeres, desde 1975 (Monk, 2020). Éste vio la luz hasta 1979, durante el XI Congreso Mundial, y para entonces ya

pués Ilamado Liga Comunista Internacionalista) y una de las facciones de la Liga Socialista). 
planteaba con claridad una hoja de ruta para el trabajo de la organización y sus diversas secciones. El texto incluía un análisis de la situación de subordinación de las mujeres, un repaso del movimiento de liberación y algunas de sus consecuencias en contextos específicos. Entre los detalles más relevantes del documento está su comprensión del correlato ideológico en la subordinación de las mujeres. Así, iba más allá de los análisis propiamente materialistas e incluía cuestiones como la relación entre las crisis económicas y las reacciones antifeministas de la clase dominante: para excluir a las mujeres de la fuerza de trabajo, se dejaba de gastar en servicios de seguridad social y se impulsaba una "ofensiva ideológica contra el mismo concepto de la igualdad y la independencia de las mujeres". En ese mismo sentido, el documento reparaba en las estrategias psicológicas y morales para la dominación del cuerpo femenino y la "racionalización" que perpetúa las ideas de que las mujeres no deben trabajar, educarse o participar de la política (Cuarta Internacional, s.f.).

El programa que se propuso la Internacional (IV Internacional) a finales de la década de 1970 para la defensa de las mujeres y su integración como parte "fundamental de la revolución mundial" era: 1. Plena igualdad política, legal y social para las mujeres; 2. Por el derecho de las mujeres a controlar sus funciones reproductivas; ${ }^{3} 3$. Por el fin de la hipocresía, la humillación y la coerción de las leyes familiares feudales y burguesas; 4 . Plena independencia económica de la mujer; 5. Igualdad de oportunidades en la educación; 6 . Reorganización de la sociedad para eliminar la esclavitud doméstica de la mujer (Cuarta Internacional, s.f.). Por otro lado, el resolutivo indicaba los ajustes que debían hacer las secciones nacionales para integrar las lógicas de género en su trabajo político cotidiano. Primero, mediante el impulso de la educación feminista hasta que ésta fuera "parte de la vida diaria de la organización"; y en segundo lugar, proponiendo la participa-

3 Esto incluía el "aborto libre y gratuito; alto a la esterilización forzosa y a cualquier otra interferencia gubernamental en el derecho de las mujeres de elegir si tener hijos y cuándo". ción de todos los militantes en las discusiones de estos temas para evitar las facciones no-mixtas que se habían empezado a multiplicar entre distintas secciones. Aunque se insistía en que estas facciones femeninas estaban "en contradicción con el carácter político del partido y con nuestros principios organizativos centralistas democráticos", al mismo tiempo, empero, se reconocía e insistía en que el feminismo de la Cuarta debía ser liderado por mujeres (Cuarta Internacional, s.f.).

Si bien esto no necesariamente se concretó a cabalidad en el trabajo cotidiano de las secciones -y el caso mexicano es un ejemplo-, la resolución habla de una reflexión autocrítica del trotskismo internacional sobre las organizaciones políticas como espacios de reproducción de las dinámicas sociales que habrían impactado en mayor o menor medida las secciones nacionales (Cuarta Internacional, 1979). ${ }^{4}$ EI PRT es un buen ejemplo. Pero lo más relevante es que se trataba de un programa que estaba completamente a tono con las luchas de la segunda ola del feminismo, que buscaba nutrir las discusiones y el trabajo del tema en las distintas secciones nacionales de la IV Internacional pero que también se inspiraba en ellas. Como se verá más adelante, el resolutivo de 1979 distinguió la situación particular de las mujeres del Tercer mundo, recogiendo así las demandas específicas de las secciones de esos países, entre las que estaba la de México, gracias a las mujeres militantes.

Sin embargo, además de la discusión que tuvo lugar a lo largo de la década en la directriz de la Internacional, el aprendizaje feminista de las trotskistas en México pasó también por la lectura de textos teóricos, que generaban otro tipo de diálogos entre militantes. En general, los grupos trotskistas dedicaban mucho tiempo a la lectura y a la

4 Esto se complementaba con una crítica a otras corrientes del movimiento obrero y su tratamiento del problema de la mujer, y la denuncia a las políticas estalinistas que habían revertido los avances de la Revolución de octubre. Asimismo decían que "las posiciones formales que han tomado los PS, frecuentemente han sido más progresistas que las de los partidos estalinistas, especialmente en relación al aborto como un derecho de la mujer". 
discusión teórica, pues la lógica de crear un partido de vanguardias implicaba una educación de cuadros formal y pronta (Mandel, 1989). ${ }^{5}$ En México, la mayoría de los jóvenes eran cooptados por el partido en las preparatorias capitalinas del Colegio de Ciencias y Humanidades $(\mathrm{CCH})$, que estableció en ellas escuelas de cuadros muy activas (Ortíz Magallón, 2020). En muchos casos los propios profesores eran ya militantes e introducían a los jóvenes a las lecturas básicas del marxismo en el salón de clases (Rodríguez Araujo, 2015: 80-81). ${ }^{6}$ Entre las lecturas a las que eran introducidas en estos circuitos, las mujeres del PRT recuerdan la prominencia de los libros de Alexandra Kollontai, sobre todo La Mujer Nueva y la Moral Sexual, de 1918 (López Rosado y Márquez Gileta, 2019: $86)^{7}$

Vale la pena detenerse en esta teórica rusa para entender cómo se podría haber formado una cultura feminista y socialista de carácter nacional, paralela a la de las redes del socialismo internacional. En la década de los setenta, la obra de Kollontai había sido reeditada y traducida al español justamente por lo que explica la feminista francesa Jacqueline Heinen: "la voluntad que tenemos de volver a apropiarnos las experiencias del pensamiento marxista en lo que concierne a la opresión de las mujeres, pero también la necesidad de hacer avanzar el debate superando los análisis realizados hasta ahora, son las razones que nos guían en la lectura crítica que intentamos efectuar de los escritos de Kollontai" (Heinen, 1979: 34). Los libros de la famosa dirigente del Zhenotdel -la organización dedicada a las mujeres dentro del Comité Central del Partido Comunista ruso-, entre 1919 y 1922, estaban dedicados tanto a las políticas públicas para

\footnotetext{
5 La lectura de Trotsky sobre las fluctuaciones del movimiento obrero lo llevaron a conceptualizar los partidos de vanguardia, que permitían mantener el trabajo constante y reaccionar frente a las condiciones para asegurar el triunfo revolucionario. Eran una parte esencial de la idea de la revolución permanente. 6 Era el caso del famoso dirigente del PRT, Alfonso Peralta, maestro en el $\mathrm{CCH}$ Oriente, asesinado en mayo de 1977

7 Elizabeth López Rosado recuerda que se impartía en la materia "Lectura de Clásicos" del CCH Oriente.
}

mejorar la vida de las obreras y las madres, como a trazar el camino de la emancipación femenina individual (Sypnowich, 1993: 290). Daba así muchos elementos para introducir el feminismo a la lucha de clases, reparando entre los distintos grados de opresión a los que están sujetas las mujeres dependiendo de su clase social. ${ }^{8}$ Kollontai fue embajadora de la URSS en México durante un breve periodo entre 1926 y 1927 y aunque sus diarios registran escasos contactos con feministas mexicanas (Ortiz, 2012), su obra sí parece haber trascendido a juzgar por la participación de una "Liga femenil Alejandra Kollontay" proveniente de Nuevo León, registrada en las memorias del Segundo Congreso de Obreras y Campesinas en 1933 (Olivé, 1991: 54). A finales de los setenta, y también en Nuevo León, un grupo de trotskistas formarían su homónima: la Brigada Alejandra KoIlontai. Urania Chavarría Decanini, militante trotskista, recuerda que la brigada organizaba "escuelas de cuadros abiertos [...] aprendíamos de la especificidad de la opresión de las mujeres, de la doble jornada de trabajo y de nuestros derechos sexuales y reproductivos" (Chavarría Decanini, 2019: 39).

A decir de algunas militantes, si bien Marx, Engels, Bebel y Kropotkin eran más que suficientes para entender las lógicas de la opresión familiar - "lo único que importaba era leerlos en clave de género" (Ortíz Magallón, 2020)-, también había lecturas contemporáneas a las que podían hacer referencia las militantes. Dasnher Monk recuerda que las feministas en las secciones de Estados Unidos, Inglaterra, Francia e Italia publicaban y discutían ampliamente "la cuestión de la mujer", y las mexicanas "devoraban" estos escritos (Monk, 2020). A través de la circulación de textos y personas que animaba la

8 Un buen ejemplo de esto es Working woman and mother (Holt, 1980), un panfleto que refleja la experiencia de la maternidad de distintas mujeres rusas y narra las historias de cuatro mujeres embarazadas hipotéticas. La esposa del dueño de la fábrica vive un embarazo cuidado y acompañado mientras que su empleada doméstica debe trabajar hasta el día de su parto. Ambas se distinguen de la trabajadora doméstica -a la que el patrón embaraza y despide-, así como de la obrera que tiene que trabajar en condiciones infrahumanas. 
IV Internacional, las mujeres que se habían integrado al trotskismo en México pudieron conocer e incluso traducir algunos de estos textos. Es el caso del trabajo de Evelyn Reed, del Socialist Workers Party, quien en una lectura inspirada en El origen de la familia, la propiedad privada y el Estado, de Engels -en donde la familia se conceptualiza como un mecanismo básico de opresión burguesa pero que también recuperaba algunas visiones propias del feminismo de la segunda ola-, explicaba la desigualdad de género a partir del sometimiento de la mujer al matrimonio, como único medio de supervivencia en la sociedad de clases. En un artículo publicado en La Internacional, el órgano del PRT, Reed explicaba cómo esta combinación de subordinaciones obligaba a las mujeres a luchar como trabajadoras, por la igualdad material, y en tanto mujeres: "para reconquistar el control de sus vidas". En ese sentido, Reed incluso hablaba del aborto como forma de "control de los [propios] procesos reproductivos", de una nueva moral sexual y familiar, y reclamaba la objetificación y la violencia contra las mujeres (Reed, 1977: 18). Junto con teóricas más clásicas, Reed se volvió una fuente teórica muy completa, que además era militante y contemporánea de las mujeres del PRT. También había otros libros disponibles para que las mujeres mexicanas hicieran su propia lectura de la teoría feminista y la implementaran en su trabajo de organización. María Elena Carrillo recuerda haber conseguido el discurso de Fidel Castro en el acto de la clausura del Segundo Congreso de La Federación de Mujeres Cubanas de 1974, así como El Poder de la Mujer de Dalla Costa y La Subversión de la Comunidad de Selma James, por ejemplo (Carrillo, 2019: 29).

Pero integrar las lecturas a las discusiones del partido implicaba otro tipo de trabajo: no era fácil probar a los camaradas varones que efectivamente había bases teóricas para explicar y combatir la desigualdad de género desde el socialismo y hacerlo con suficiente autoridad para ser tomadas en serio. Las militantes recuerdan que se dificultaba sobre todo porque las interesadas en el feminismo eran todas "compañeras de base", como explica Monk, lo que les implicaba ceñirse a las jerarquías y hacer el trabajo doble de convencer a compañeros y dirigentes (Monk, 2020). Las formas en que el partido reproducía la desigualdad e ignoraba a las compañeras también necesitaban ser denunciadas constantemente. $Y$ en la construcción de lo que aspiraba a ser un sentido común compartido por los militantes, hubo voces particularmente insistentes y elocuentes. Éste parece haber sido el caso de Ana María Flores, según recuerda Rosario Ortíz Magallón, una militante más joven que el promedio de sus compañeras en esos primeros años del PRT que veía a esta mujer con admiración (Ortíz Magallón, 2020). También estaba Susana Vidales, quien incluso escribió en 1977 un texto sobre los residuos "sexistas y opresores" en el PRT y de la importancia de educar a los militantes sobre "la opresión que sufre la mujer en sociedad y por tanto, de su desigualdad objetiva al interior de los partidos políticos" (Vidales, 1977: 8-9, 13) y la propia Heather Monk. Estas prácticas y dinámicas parecen haber sido recurrentes en las distintas secciones del mundo, a juzgar por el documento del XI Congreso de un par de años más tarde (Cuarta Internacional, s.f.). Sin embargo, las formas concretas que tomaban tanto la discriminación como su denuncia variaban según cada contexto.

Por otro lado, también es cierto que la forma de organizarse dentro del PRT fue lo que permitió a las mujeres militantes trabajar por sus objetivos. Como resultado del trauma que había dejado el sectarismo de las décadas anteriores (Rodríguez Araujo recuerda la broma: "donde hay dos trotskistas éstos se dividen") y, de acuerdo con los principios democráticos del trotskismo, los estatutos del Partido Revolucionario de los Trabajadores dejaban claro que todos sus miembros tenían derecho de reunirse en "tendencias" (Rodríguez Araujo, 2015: 73). Estas tendencias podían "presentar sus posiciones a [el resto de] los militantes de la organización durante los períodos de discusión", crear programas y visitar las células 
que conformaban el trabajo cotidiano del partido (Estatutos del PRT, 1976 en Oranday Dávila, 2015: 340). A decir de las mujeres, ésta fue la estrategia que utilizaron para avanzar los temas de género, reuniéndose sólo ellas; de ahí que el documento de la IV Internacional hablara de los grupos no-mixtos con suspicacia pero también cierto realismo (Cuarta Internacional, s.f.). Y también les permitió coordinarse con la comisión especializada que se dedicaba sobre todo a la elaboración de folletos y crear publicaciones sobre temas como "la sexualidad, el aborto, la familia, el trabajo doméstico, la situación de la mujer campesina y obrera" (Vidales, 1977: 11; Monk, 2020). Finalmente, y más importante aún, este andamiaje institucional permitió que el partido extendiera su actividad feminista fuera de sus filas. Según la militante Urania Chavarría, "este principio organizativo sería replicado en los movimientos en donde participábamos y teníamos influencia", incluidos los sindicatos y universidades (Chavarría Decanini, 2019: 39), con lo cual este feminismo trotskista se volvía una opción de movilización fuera de las discusiones y organización del partido. ${ }^{9}$

\section{La segunda ola en México}

Aunque las dinámicas propias del trotskismo efectivamente permitieron y nutrieron la lucha de sus militantes mujeres en todo el mundo, el movimiento por la liberación de la mujer en México jugó un papel innegable en la toma de conciencia y formas de organización de las trotskistas mexicanas. Para el momento de la formación del PRT, el feminismo llevaba más de un lustro activo en el país. Los grupos y temas que surgieron de este contexto fueron determinantes para la forma que cobraron algunas demandas feministas en el PRT, su trabajo político, e incluso para la contribución de la sección mexicana a la resolución del XI Congreso.

El feminismo llegó a México en la década de 1970 por influencia del movimiento orga-

9 Aunque Rosario Ortíz Magallón recuerda que, por lo menos en el mundo sindical, estas ideas tardaron mucho en introducirse y nunca se aceptaron realmente (Ortíz Magallón, 2020). nizado en Estados Unidos, según recuerdan sus primeras exponentes (Lamas, 2020). Sin embargo, la agenda de la liberación de la mujer pronto adquirió tintes nacionales que adoptaron las propias trotskistas, no obstante su afán internacionalista.

Buena muestra de los ejes sobre los que se fue articulando un feminismo vernáculo es el artículo de Rosario Castellanos de 1970 titulado "Casandra de huarache: la liberación de la mujer, aquí", en el que la escritora denunciaba la tendencia latinoamericana a delegar a la mujer el trabajo doméstico y los cuidados, lo que no sólo creaba una doble opresión para las mujeres más pobres, sino que evitaba la masificación del movimiento (Reyes, 2006: 561-564). Demandas similares, resultado de estos intentos por conceptualizar el tipo de opresión que vivían las mujeres en un país como México se condensaron bien en las manifestaciones alrededor de la Conferencia Mundial por el Año Internacional de la Mujer (AIM), de la Organización de las Naciones Unidas, que tuvo su sede justamente en México, en 1975 (Olcott, 2017).

Ese año, varios grupos feministas, entre los que estaban las trotskistas, se reunieron en el efímero Frente de Mujeres contra el Año Internacional de la Mujer. Como declaran sus distintos comunicados, las feministas reunidas en este frente veían en esta conferencia una forma de despolitización del movimiento feminista $y$, retomando los principios de Igualdad, Desarrollo y Paz impulsados por la ONU, declaraban: "No queremos igualdad de condiciones para ser explotadas de la misma manera que los hombres. No queremos un desarrollo que perpetúe la desigualdad económica, racial y sexual. No queremos una paz que sólo signifique la estabilidad del sistema actual" (Movimiento de Liberación de la Mujer, 1975a). En distintos panfletos y manifiestos plantearon que cualquier cambio sustancial a la situación de la mujer necesitaba del fin del sistema capitalista e insistieron en las diferencias entre las mujeres del Tercer mundo y sus contrapartes de los países desarrollados, sobre todo en cuanto al tema del traba- 
jo doméstico y el aborto. En boca de estas feministas, la maternidad era una cuestión de clase, pues ésta, incluso cuando era elegida, no era realmente libre si "no hay dinero suficiente para criar [a los hijos], una vivienda segura, ni tiempo para disfrutarlos", sentenciaban (Movimiento de Liberación de la Mujer, 1975b y 1975c). Estos serían temas prominentes en las publicaciones del PRT los años posteriores.

El Frente contra el Año Internacional de la Mujer fue liderado por las mujeres del Movimiento de Liberación de la Mujer, un grupo de feministas de clase media que se autoidentificaban con la izquierda pero que no militaban en organizaciones partidistas. Con él estaba el Grupo Liberación Femenina y, notablemente, las mujeres trotskistas, tanto del Grupo Comunista Internacionalista (GCl) como de la Liga Socialista (LS), antecedentes inmediatos del PRT. Como recuerda Josefina Chávez, desde 1971 el GCl ya tenía una organización interna de mujeres: "para la formación feminista, se elaboraban artículos para la prensa partidaria y se diseñaban las tácticas y orientaciones para impulsar el desarrollo de un movimiento feminista con perspectiva clasista" (Chávez Rodríguez, 2019: 51). Esta colaboración revela que, antes de que el PRT unificara a las corrientes trotskistas, las mujeres de ambos grupos ya participaban juntas en actividades por la liberación de la mujer, algo que ayudaría a explicar la pronta organización de trabajo feminista dentro del partido. De hecho, los dos órganos de difusión, El socialista de la LS y Rojo del GCl, publicaron textos a propósito del Año Internacional de la Mujer en los que queda de manifiesto la complementariedad de sus posturas. En el primero, María Luisa Hernández denunciaba el carácter capitalista que la ONU le daba a la liberación de la mujer, en el mismo sentido de lo expuesto por el Frente, y proponía un programa con elementos que serían retomados por el posterior PRT: total igualdad política y económica para las mujeres, independencia económica, control de las propias funciones reproductivas, igualdad de oportunidades y "libertad de la esclavi- tud doméstica” (Hernández, 1975). Un par de meses después, ya en plena Conferencia, Leonor Torres escribió sobre las raíces históricas del movimiento de mujeres y analizó los retos que enfrentaba entre "los revolucionarios" mexicanos, que podían equivocarse al interpretarlo como "pequeñoburgués" (Torres, 1975).

Pero este evento de 1975 es muestra de algo más: la colaboración que las trotskistas mantendrían con las feministas fuera del mundo partidista, de donde adquirieron ideas, alianzas y otros espacios para seguir construyendo sus causas. Para algunas mujeres militantes, la cercanía con grupos como el MLM fue fundamental para profundizar en su feminismo de formas distintas al partido. Por ejemplo, Guadalupe Hernández relata que el contacto con este grupo fue indispensable en su formación, entre otras cosas porque se integraría a trabajar con algunas de estas mujeres en la administración de la revista feminista FEM, fundada en 1976 y especializada en temas de feminismo y la mujer (Hernández López, 2019: 96). Pero la cercanía incluso trascendió al trabajo feminista. Como recuerda Heather Dashner Monk, hubo un tiempo en que, para evitar la represión del gobierno, el periódico del PRT se hizo desde la casa de Marta Lamas, una feminista muy activa del MLM, que se solidarizó con el partido a partir del trabajo realizado en conjunto con algunas de sus militantes (Monk, 2020). En ese sentido, los vínculos con otras organizaciones de mujeres parecían hacer del grupo de feministas dentro del PRT una agrupación con un cierto poder social distintivo, que seguramente les ayudaba a negociar con otros miembros de la organización las causas que impulsar.

Finalmente, la labor de cooperación inaugurada en 1975 se repetiría en otro frente más adelante. El Frente Nacional por la Liberación y los Derechos de las Mujeres (FNALIDM) reunió en 1979 a una coalición de feministas, aún más grande que su predecesora durante el Año Internacional de la Mujer, alrededor de una plataforma muy amplia que eventualmente concentró sus esfuerzos en la lucha por el aborto. A esta 
coalición incluso se integraron las comunistas del PCM, lo que significó una colaboración sin precedentes entre el trotskismo y el comunismo asociado a Moscú. Cierto es que las mujeres del PC habían empezado su propio proceso en el trabajo de género. Esto lo prueban algunos eventos y conferencias durante la primera emisión del Festival "Oposición" (PCM, 1977: 6). ${ }^{10}$ Sin embargo, según Monk, las ideas del feminismo entraron al PC después de que Amalia García fuera su representante en el FNALIDM, y no producto de las propias reflexiones del PC (Monk, 2020). Esto es algo que García confirma, aunque atribuye su feminismo al clima general de la época y a la influencia de camaradas en otras partes del mundo, como las francesas de la frase citada al principio de este artículo (García, 2020). Algo que, sin embargo, es muy notorio, es que el feminismo que se introdujo al Partido Comunista no llevó a una reflexión amplia sobre la sexualidad, que eventualmente incluyera a la homosexualidad, a diferencia de lo que sí sucedió en el PRT. Y esto impediría la colaboración ulterior entre comunistas y trotskistas, según recuerda la militante del PRT, Susana Vidales (Vidales, 2019: 124).

\section{Las mujeres en la agenda del PRT}

Esta mezcla de referentes tanto teóricos como políticos tuvo repercusiones en el trabajo concreto del PRT alrededor de la liberación de las mujeres en sus primeros años de existencia. Estos son patentes en los textos publicados en sus órganos de comunicación, pero también en las oportunidades que se dieron a las mujeres del PRT de participar en puestos directivos y de elección popular.

A tono con la IV Internacional, el PRT concebía a las mujeres como parte de la clase obrera. Ésta era una concepción que se basaba sobre todo en el trabajo doméstico pero que, a diferencia de lo que proponía la

10 El "Programa general del Primer Festival de Oposición" anunció una mesa redonda con el título "Sobre la liberación femenina". Y en años sucesivos habría eventos similares, con la participación de las propias comunistas como ponentes.
IV -que era "liberar a las mujeres del trabajo doméstico", socializando las actividades de éste-, el PRT se concentró en las mujeres que realizaban trabajo doméstico para terceros. Siguiendo el consenso del feminismo mexicano que se estableció desde principios de la década, como se ha visto, el PRT tenía muy claro que la situación de las trabajadoras domésticas en países como México se distinguía por la doble precarización de la que eran objeto. Para combatir esto, en su declaración del Comité Central de 1977, el partido se propuso nada menos que la sindicalización de las trabajadoras domésticas (PRT, 1977b: 81). Pero algo que es notorio es que esta reflexión de clase no llevaba al partido a caer en el viejo tropo de las mujeres de clase media como parte de la burguesía que combatir. En el contexto de la crisis económica, el PRT veía en las amas de casa "un sector con grandes potencialidades y una profunda radicalidad que hasta ahora se ha quedado sin posibilidades de expresión y organización", un aliado indiscutible para luchar contra la carestía. Por ello, se insistía en promover la organización entre estas mujeres, siguiendo la propia estructura de otros comités de lucha: asociarlas "en cada barrio, cada sindicato” (PRT, 1977b: 81). Así, a un año de fundado el PRT, las mujeres -en su variedad-aparecían como un sujeto susceptible de ser politizado por las causas de la mayoría precarizada. Era una forma muy original de entender a este sector, cuyas estrategias de integración ameritarían investigarse a profundidad.

Asimismo, en el partido las mujeres eran consideradas tan importantes para la lucha "multisectorial" como los hombres. Según declaraba el Comité Central apenas fundado el PRT, la revolución requería un programa, pero también "una organización y una vanguardia de hombres y mujeres comprometidos con los trabajadores, con su época y con el destino de la humanidad, que es la liberación de todas las cadenas de opresión que el capitalismo ha eslabonado y reforzado cruelmente" (PRT, 1977b: 82). Esto se reflejó en la participación de las mujeres militantes en los órganos directivos, en las 
revistas y, eventualmente, en las campañas políticas, cuya cúspide es la famosa participación de Rosario Ibarra de Piedra como candidata a la presidencia por parte del PRT en 1982 y 1988. " Según explica Josefina Chávez Rodríguez, la participación se concretó, entre otras cosas, a través de una política de acciones afirmativas, "adoptada de los movimientos por los derechos civiles en Estados Unidos", inédita en la izquierda nacional (Chávez Rodríguez, 2019: 54). Para las elecciones de 1982, las primeras en las que los partidos de izquierda pudieron participar tras la reforma de 1977, el PRT presentó a un $27 \%$ de mujeres como candidatas a senadoras. Para las elecciones de 1988 el número aumentó a 38\%. Para las diputadas estas cifras fueron de $18 \%$ y $32 \%$, respectivamente. En ambos casos rebasó por mucho la proporción de mujeres con relación al resto de los partidos políticos (PRT, Bandera Socialista, 1982: 8, en Oranday Dávila, 2015: 247).

Pero hay indicios de liderazgos de mujeres impulsados por el PRT incluso antes de obtener el registro electoral. Éste es el caso de la candidatura de Josefina Oseguera Parra en las elecciones municipales de Colima de 1976, una candidatura simbólica, dada la ilegalidad del partido, pero que buscaba avanzar la organización de la clase proletaria del estado a partir de una plataforma política concreta. La propaganda explicaba: "el voto que hoy se pierde es una identificación de intereses de clase" (PRT, 1976: 1). Sin embargo, el hecho de que se hiciera mediante la figura de una mujer y con una plataforma que, entre otras cosas, se manifestaba explícitamente por la liberación de las mujeres, es sintomático de las posibilidades políticas que tenían las mujeres dentro del trotskismo. Cabe decir que, en este caso, aunque se hablaba de la importancia de contar con métodos de control de la natalidad gratuitos, no se mencionaba al aborto, lo cual indica que el partido probablemente ajustaba en alguna medida sus propuestas, considerando también el conservadurismo de algu-

11 Cabe recordar que Ibarra no era militante del PRT y, sin embargo, se volvió una dirigente muy importante de la mano del partido gracias al trabajo conjunto en el Frente Nacional Contra la Represión. nos de los sectores a los que se dirigía (PRT, 1976: 7).

Pese a todo, esta promoción de las mujeres a puestos de poder no fue la regla general. Y en algunos sectores era más difícil avanzar la causa que en otros; por ejemplo, en el sindicalismo. Rosario Ortíz Magallón recuerda que todas aquellas victorias teóricas en el seno de las asambleas o las discusiones del PRT mostraban pronto sus límites en las comisiones dedicadas al trabajo sindical. "Si lo que se estaba discutiendo era un aumento salarial, los compañeros veían como una desviación no prioritaria cualquier cosa que tuviera que ver con los derechos de las mujeres" (Ortíz Magallón, 2020). Esto resultaba en la falta de interés e involucramiento de las mujeres del partido en estos temas en la misma dimensión que los hombres. Y con todo, se insistió en que "para involucrar a más camaradas en la orientación del trabajo en los sectores y la formación teórica, era necesaria otra instancia", y así se creó el Colectivo (Interno) de Mujeres que muy pronto se dedicó a planear, por ejemplo, una

escuela de cuadros, de pláticas en [la Universidad de] Chapingo, trabajo entre bancarias, contactos con mujeres de Tlaxcala, una manifestación de los trabajadores electricistas de la Tendencia Democrática, pláticas y círculos en las Casas de Estudiantes (especialmente en Tlaxcala); y la propuesta de movilizar a las esposas de los huelguistas de la empresa Dina (Monk, 2019: 68).

Pese a la demarcación local y nacional, estas ideas y activismo siempre tenían en mente las aspiraciones internacionalistas propias del trotskismo. Las mujeres del PRT no se conformaron con trabajar para la escena mexicana, sobre todo en vista de que la experiencia nacional podía contribuir a conceptualizar mejor los retos que enfrentaban las mujeres en el globo. Cuando las mexicanas recibieron el borrador del resolutivo sobre la situación de la mujer previsto para discutirse en el XI Congreso de la IV Internacional, encontraron que el texto no consideraba de ningún modo la particularidad de la 
situación de las mujeres en el Tercer mundo. Las mexicanas se dispusieron entonces a escribir una propuesta que enviaron a la IV, que fue retomada e incluida, con modificaciones e información sobre Asia y África, en el documento final. Heather Dashner Monk incluso viajó a Nueva York para participar de la última redacción (Monk, 2020). Así, el resolutivo integró un análisis sobre los países "coloniales" y "semicoloniales" que denunciaba que:

Para las mujeres en el mundo colonial y semicolonial la penetración de la economía capitalista de mercado tiene un impacto contradictorio: por una parte introduce nuevas relaciones económicas que comienzan a crear la base para que las mujeres superen su opresión de siglos; pero por otra parte toma y utiliza las tradiciones arcaicas, los códigos religiosos, y los prejuicios contra las mujeres reforzándolos inicialmente con nuevas formas de discriminación y sobreexplotación (Cuarta Internacional, s.f.).

Asimismo, incluía reflexiones sobre la migración campo-ciudad, la religión, la falta de educación y la violencia física, las restricciones al control de la natalidad, la esterilización forzada, entre otros temas reconocibles en las discusiones de las feministas mexicanas. El feminismo del PRT, con sus múltiples fuentes de inspiración teóricas y a partir de la realidad política y social mexicana, llegaba a fines de la década de 1970 con suficiente precisión y fuerza, como para tener algo que aportar al centro directriz de la lucha trotskista internacional. Dentro de las fronteras nacionales, tan sólo un par de años después este mismo trabajo se vería reflejado en la primera candidatura de una mujer a la presidencia de México.

\section{Conclusiones y caminos abiertos}

Esta investigación se preguntó por el origen y los alcances teóricos y políticos de ciertas ideas sobre la liberación de la mujer, identificables en algunos de los primeros textos programáticos del Partido Revolucionario de los Trabajadores, fundado en 1976. La presencia de estas ideas llama la atención dado el rechazo de buena parte de las izquierdas marxistas a los derechos de sectores específicos, por considerar prioritaria a la lucha de clases. Con base en testimonios de mujeres militantes del PRT, así como programas y publicaciones del partido, aquí se ha analizado la introducción del feminismo en los primeros años de existencia de esta agrupación, sus fuentes y algunos de sus resultados concretos. Como se ha podido ver, las ideas sobre la liberación de la mujer adoptadas por el partido provinieron de diversas fuentes. Las movilizaciones feministas de la década de 1960 y 1970 impactaron en diversas partes del mundo y, frente a ellas, la IV Internacional no se mantuvo incólume. Dentro del órgano central y entre las redes del trotskismo, se desató un proceso paralelo de reflexión e integración de las ideas sobre la liberación de la mujer. Para las trotskistas mexicanas, fue igualmente relevante la lectura "en clave de género" que hicieron de teóricos y teóricas de la izquierda marxista, clásicos y contemporáneos.

Sin embargo, entre las ideas que adoptó como suyas el PRT se identifican también las preocupaciones y temas propios del feminismo "de la segunda ola" en México, como el aborto y el trabajo doméstico que, de hecho, serán contribuciones de las trotskistas mexicanas a las resoluciones del XI Congreso de la IV Internacional. Como se vio, diversas acciones emprendidas por mujeres feministas en México vincularon a mujeres militantes del Grupo Comunista Internacionalista ( $\mathrm{GCl}$ ) y de la Liga Socialista (LS), incluso antes de su unificación en PRT. Las dinámicas de la lucha feminista fuera del trotskismo dieron a estas mujeres, y al propio partido, aliadas importantes.

Las consecuencias de esto son claras en ciertas líneas programáticas adoptadas por el PRT, así como en la introducción sostenida de mujeres a candidaturas de elección popular y en puestos de responsabilidad del partido. Como se vio, nada de esto fue fácil. Las militantes admiten que al interior del partido encontraron diversos obstáculos 
para convencer a sus compañeros sobre la relevancia del trabajo para y con mujeres. No obstante, dinámicas propias del trotskismo también les permitieron a las mujeres organizarse, y más importante aún, llevar su actividad feminista fuera de sus filas.

La experiencia del trotskismo en la lucha por la liberación de la mujer es susceptible de leerse a partir de la influencia de la nueva izquierda. La incorporación teórica de sujetos distintos al proletariado en abstracto es reconocible en el partido, algo que seguramente se explica por la cercanía de muchas de las secciones trotskistas -entre las que estaba la mexicana- al movimiento estudiantil y a los grupos feministas (Hughes, 2012). Sin embargo, como se ha visto, buena parte de la forma que tomaron las discusiones y agendas sobre la mujer en el PRT se explican por las dinámicas propias del trotskismo: la lógica de vanguardia, la educación teórica, el respeto a las agendas de cada sección y cada tendencia, así como el apoyo y redes internacionales. Por su parte, los grupos de mujeres trotskistas en México tenían experiencia trabajando fuera del espacio del partido, en colaboración con otros colectivos feministas desde principios de la década de 1970. Esto les permitió profundizar en su conocimiento teórico y sus experiencias políticas, logrando incorporar propuestas específicas para entender y atajar la situación de la mujer en México que pronto fueron retomadas como parte de la agenda partidista central, e incluso en la propia dirigencia de la IV Internacional.

Pese a los esfuerzos, en general las militantes reconocen que el partido, si bien integró en sus agendas y planes al feminismo, no siempre lo hizo en la medida, ni con la diligencia que ellas hubieran querido. De hecho, en algunos de los textos teóricos de la misma época hay contradicciones evidentes que sería importante analizar con detalle. Por ejemplo, la declaración de que el PRT, en la búsqueda por el modelo de frente único -contra el de la unidad de la izquierda [al estilo de la Unidad Popular chilena]- debía "abandonar todo tipo de veleidades en las alianzas multiclasistas, que los partidos reformadores impulsan en todas partes, y concretarse a la búsqueda de una unidad con objetivos revolucionarios y de clase, que plantea el rechazo de las alianzas con las burguesías progresistas" (Rodríguez Araujo, 1980: 216). O el matiz que señalaba que el partido: "aunque por principio participa e influye en todos los movimientos cotidianos del proletariado en que sus recursos le permiten intervenir, no se diluye en ellos [...] coloca todas estas luchas como pasos, puentes o etapas transitorias que deben eslabonarse con la victoria total contra la burguesía: la revolución socialista" (PRT, 1977c). En estas contradicciones se adivina la pervivencia de algunos dogmas del marxismo, que muy seguido resultaban en generalizaciones inútiles para entender realidades intrincadas, como la mexicana, y que seguramente influyeron en las posibilidades del trotskismo para transformar la realidad.

En ese sentido, queda por investigarse la relación que guarda el desarrollo del PRT con las directrices generales de la IV Internacional más allá del feminismo. Todo el periodo revisado aquí se inscribe en un momento de discusiones álgidas sobre cuál debía ser la política del trotskismo para América Latina. Tras el triunfo de la Revolución cubana y sobre todo en vista de las promesas de la Revolución boliviana, en el IX Congreso Mundial se había convenido que la mejor estrategia para lograr la revolución en la región era efectivamente la guerra de guerrillas. Aunque ésta fue la "opinión mayoritaria”, encabezada por el italiano Livio Maitan, las críticas se hicieron oír muy pronto, inclusive de parte de las secciones latinoamericanas. Desde 1970, Joseph Hansen, del Socialist Workers Party estadounidense, recogió muchas de ellas y anunció el fracaso de la estrategia (Hansen, 1970: 6). Pese a eso, en el X Congreso Mundial de 1974 la línea de guerra de guerrillas fue profundizada y asumida como directriz, no sólo para América Latina, sino para el mundo entero, según reportaron los delegados argentinos que asistieron a ese congreso ("Ernesto", 1974). No sería sino hasta 1977 en que la IV Internacional empezara a expresar abiertamente cier- 
tas autocríticas sobre la estrategia, y para el Congreso Mundial de 1979, a la par de la resolución sobre la liberación de las mujeres, hubo también una resolución sobre América Latina. En ella, la IV Internacional asumía su error y cambiaba su orientación para el trabajo en la región (Marxists Internet Archive, 2005). Todo esto fue registrado por Méxi$c o$, en donde nunca se aceptó realmente la idea de las guerrillas (PST, 1974; Ríos, 1978: 8-10).12 Sin embargo, Rosario Ortíz Magallón cuenta que desde el CGI había un grupo radicalizado del que ella formaba parte y, lo que es más, le atribuye a su participación en éste, y a su enfoque en la lucha armada, su particular llegada tardía al feminismo (Ortíz Magallón, 2020).

La experiencia personal de Ortiz hace eco de la transformación de agenda de la IV Internacional. Y si bien en ésta los temas de la guerrilla y las mujeres no parecen haber sido excluyentes, valdría la pena analizar los problemas de teoría revolucionaria que esto le planteó a las distintas secciones. Por lo pronto, hay una pista paradójica en el hecho de que el PST de Argentina, reconocidamente antiguerrillero, reportara, tras el X Congreso de marzo de 1974, que consideraba "exagerada" la importancia que la IV Internacional le empezaba a dar al movimiento de las mujeres mientras se ignoraba al movimiento campesino en países como India o Bolivia, que era "tanto o más importante" ("Ernesto", 1974). Visto desde la perspectiva del eterno dilema de la jerarquización de causas, el caso mexicano y su búsqueda por una revolución con agentes múltiples y definidos más allá de la abstracción del proletariado, es aún más interesante y abre múltiples preguntas sobre la articulación del trotskismo en la región y la relación de los distintos países con la dirigencia central.

12 En 1974, la Liga Socialista mandó un mensaje al Partido Socialista de los Trabajadores argentino: Io consideraban "la inspiración para llevar a cabo la estrategia leninista", y que debía ser un modelo para América Latina. Esto es importante porque quiere decir que México se identificaba con una de las secciones más críticas de la estrategia de guerrillas, la liderada por Nahuel Moreno en Argentina.
Finalmente, en cuanto a la experiencia de la militancia en concreto, cabe decir que el trabajo feminista avanzó toda una serie de temas sociales y culturales dentro del Partido Revolucionario de los Trabajadores en México, del que aquí hemos visto apenas algunas características. Como escribió Susana Vidales en 1977, muchas de las discusiones que motivó el feminismo dentro de las filas del partido "parecían vedadas hasta para las organizaciones de izquierda”. Era el caso de la vida cotidiana del militante, la homosexualidad, la drogadicción (Vidales, 1977: 12). Es probablemente aquí, en las experiencias de los militantes, moldeadas por el día a día de organizaciones partidistas que existían a caballo entre las expectativas de la lucha de clases global y las posibilidades locales, en donde el alcance de las agendas concretas, como la liberación de la mujer, adquieren su verdadera relevancia política.

\section{Fuentes consultadas}

\section{Archivos}

"Ernesto" (1974), "Informe sobre el Décimo Congreso de la IV Internacional", marzo, documento de trabajo. Archivo Fundación Pluma Primer Período: del GOM al PST inclusive, Documentos entre 1969 y 1975, <https://cutt.ly/jInJsVU>, 10 de enero de 2022

Marxists Internet Archive (2005), "Declaration of the International Majority Tendency (julio 1977)", Toward a History of the Fourth International, Marxist Internet Archive, <https://cutt.ly/ilnJvlv>, 25 de noviembre de 2020.

Movimiento de Liberación de la Mujer (1975a), "Boletín de Prensa sobre el Año Internacional de la Mujer", marzo, boletín de prensa. Archivo personal de Marta Lamas, Ciudad de México.

Movimiento de Liberación de la Mujer (1975b), “A qué se debe que en el Año Internacional de la Mujer no se hable de...", circa junio, boletín de prensa. Ar- 
chivo personal de Marta Lamas, Ciudad de México.

Movimiento de Liberación de la Mujer (1975c), "Declaración por la liberación de presas en Italia", México D.F., 14 de febrero, comunicado. Archivo personal de Marta Lamas, Ciudad de México.

PST (Partido Socialista de los Trabajadores) (1974) "Mensaje de los compañeros mexicanos con motivo del Congreso", 28 de octubre, documento de trabajo. Archivo Fundación Pluma, Argentina, Documentos de la Fundación Pluma Primer Período: del GOM al PST inclusive, Documentos entre 1969 y 1975, <https://cutt. ly/1InJI7S>, 29 de noviembre de 2020 .

Vidales, Susana (1977), "La mujer: partidos y sindicatos". Ponencia presentada por el Partido Mexciano de los Trabajadores al Primer Simposio Mexicano-Latinoamericano de Investigación de la Mujer, México, 7, 8 y 9 de noviembre, documento de trabajo. Biblioteca del Colegio de México.

\section{Referencias}

Bartra, Eli (1999), "El movimiento feminista en México y su vínculo con la academia", Revista de Estudios de Género. La ventana, núm. 10, Guadalajara, Universidad de Guadalajara, pp. 214-234.

Carrillo, María Elena (2019), "El feminismo y mi experiencia en la lucha de clases", en Beatriz López Rosado y Ángeles Márquez Gileta (eds.), Feministas Trotskistas, Ciudad de México, Nova Gráficos, pp. 28-35.

GCl (Grupo Comunista Internacionalista) (1971), "Revolucionarios: a construir la vanguardia leninista”, La Internacional, núm. 17, Ciudad de México.

Chavarría Decanini, Urania (2019), "Yo trotska", en Beatriz López Rosado y Ángeles Márquez Gileta (eds.), Feministas Trotskistas, Ciudad de México, Nova Gráficos, pp. 32-43.
Chávez Rodríguez, Josefina (2019), "El Feminismo, el PRT: mi experiencia", en Beatriz López Rosado y Ángeles Márquez Gileta (eds.), Feministas Trotskistas, Ciudad de México, Nova Gráficos, pp. 44-59.

Comer, Clémentine (2018), "Ludivine Bantigny, Fanny Bugnon et Fanny Gallot (dir.), 'Prolétaires de tous les pays, qui lave vos chaussettes?' Le genre de l'engagement dans les années 1968", Clio. Femmes, genre, histoire, núm. 48, Rennes, Presses universitaires de Rennes, <https://bit.ly/3mQfQyl>, 25 de noviembre de 2020.

Cuarta Internacional (1979), "La revolución socialista y la lucha por la liberación de la mujer", XI Congreso Mundial, Cuarta Internacional, <https://bit.ly/3CPfOYP>, 25 de noviembre de 2020.

Hansen, Joseph, (1970), "A contribution to the discussion of revolutionary strategy in Latin America", International Information Bulletin, núm. 6, Archivo Marxists Internet Archive, <https://bit. ly/3q7zO5v>, 9 de octubre de 2021.

Heinen, Jacqueline (1979), "Introducción”, en Alexandra Kollontai, Sobre la liberación de la mujer (Seminario de Leningrado de 1921), trad. de Michèle Lenard, Barcelona, Fontamara.

Hernández, María Luisa (1975), "Desde la ONU. El Año Internacional de la Mujer", El Socialista, Ciudad de México.

Hernández López, Guadalupe (2019), "De cómo llegué al feminismo y al socialismo", en Beatriz López Rosado y Ángeles Márquez Gileta (eds.), Feministas Trotskistas, Ciudad de México, Nova Gráficos, pp. 92-97.

Holt, Alix (1980), Selected Writings of Alexandra Kollontai, Londres, Norton.

Honeycutt, Karen (1976), "Clara Zetkin: A Socialist Approach to the Problem of Woman's Oppression", Feminist Studies, 3 (4), College Park, University of 
Maryland, pp. 131-144, <https://bit.ly/ 3bHCBPj>, 6 de septiembre de 2021.

Hughes, Celia (2012), "Young Socialist Men in 1960s Britain: Subjectivity and Sociability", History Workshop Journal, núm. 73, Londres, Oxford University Press, pp. 170-192.

Lamas, Marta (2020), "El revolcón de mi primer año", Nexos, Ciudad de México, <https://bit.ly/3o00YLR>, 6 de septiembre de 2021.

López Rosado, Beatriz y Márquez Gileta, Ángeles (eds.) (2019), Feministas Trotskistas, Ciudad de México, Nova Gráficos.

Lau Jaiven, Ana (2006), "Feminismo en México", en Nathalie Lebon y Elizabeth Maier (coords.), De lo privado a lo público: 30 años de lucha ciudadana de las mujeres en América Latina, Ciudad de México, Siglo XXI/UNIFEM/LASA, pp. 181-194.

Mandel, Ernest (1989), “Trotsky's conception of self-organisation and the vanguard party", Marxists' Internet Archive, 2007, <https://bit.ly/3GUtjxw>, 25 de noviembre de 2020.

Olivé, Natura (1991), "Las mujeres en el partido comunista mexicano. Los años treinta", tesis de maestría en Historia, Escuela Nacional de Antropología e Historia, Ciudad de México.

Olcott, Jocelyn (2017), International Women's Year, Oxford, Oxford University Press.

Ortiz, Rina (2012), Alexandra Kollontai en México: diario y otros documentos, Xalapa, Universidad Veracruzana.

Oranday Dávila, María Stella (2015), La revolución de la libertad: el PRT y la lucha de las mujeres mexicanas por sus derechos, Ciudad de México, Estampa Artes Gráficas.

PCM (Partido Comunista Mexicano) (1977), "La liberación de la mujer, simultánea a la lucha de clases", Oposición. Órgano del Comité Central del Partido Comunista Mexicano, 30 de abril, Ciudad de México, PCM.

PRT (Partido Revolucionario de los Trabajadores) (1976), "Plataforma electoral del Partido Revolucionario de los Trabajadores", noviembre, Ciudad de México, PRT.

PRT (Partido Revolucionario de los Trabajadores) (1977a), "Documentos del Primer Congreso Extraordinario del PRT", La Internacional, núms. 5 y 6, Ciudad de México, PRT.

PRT (Partido Revolucionario de los Trabajadores) (1977b), "Declaración del C.C. del PRT", La Internacional, núm. 1, Ciudad de México, PRT.

PRT (Partido Revolucionario de los Trabajadores) (1977c), ¿Qué es el PRT?, Folletos Bandera Socialista, núm. 9, México, PRT.

Reed, Evelyn (1977), "Cómo perdieron las mujeres su destino y cómo pueden reconquistarlo", La Internacional, núm. 3, Ciudad de México, pp. 11-13.

Reyes, Andrea (2006), Mujer de palabras. Artículos rescatados de Rosario CasteIlanos, t. II, Ciudad de México, Conaculta.

Ríos, Alfonso (1978), "Editorial. XL Aniversario de la IV Internacional", La Internacional, núm. 7, Ciudad de México, pp. 3-11.

Torres, Leonor (1975), "A propósito de la liberación de la mujer", Rojo. Periódico de Acción Comunista, núm. 5, Ciudad de México, pp. 3-5.

Rodríguez Araujo, Octavio (2015). "El trotskismo", Las izquierdas en México, Ciudad de México, Orfila, pp. 65-91.

Rodríguez Araujo, Octavio (1980), La reforma política y los partidos en México, Ciudad de México, Siglo XXI. 
Sypnowich, Christine (1993), "Alexandra KoIlontai and the Fate of bolshevik feminism", Labour/Le Travail, 32, Edmonton, Canadian Committee on Labour History, pp. 287-296.

Vidales, Susana (2019), "Una trotska feminista”, en Beatriz López Rosado y Ángeles Márquez Gileta (eds.), Feministas Trotskistas, México, Nova Gráficos, pp. 118-127.

\section{Entrevistas}

García, Amalia (2020), "Entrevista con Amalia García”, entrevistada por Ana Sofía Rodríguez Everaert, [Zoom], Ciudad de México, 1 de octubre de 2020.

Ortíz Magallón, Rosario (2020), "Entrevista con Rosario Ortíz Magallón”, entrevistada por Ana Sofía Rodríguez Everaert [Zoom], Ciudad de México-Bruselas, 19 de noviembre de 2020.

Monk, Heather Dashner (2020), "Entrevista con Heather Dashner Monk", entrevistada por Ana Sofía Rodríguez Everaert, [Zoom], Ciudad de México-Bruselas, 28 de noviembre de 2020.

Recibido: 1 de octubre de 2021. Aceptado: 10 de enero de 2022. Publicado: 24 de enero de 2022.

\section{Ana Sofía Rodríguez Everaert}

Es licenciada en Historia por la Universidad Nacional Autónoma de México y doctoranda en el Centro de Estudios Históricos de El Colegio de México. Su línea de investigación actual es el discurso de los derechos humanos en México a finales del siglo XX. Entre sus más recientes publicaciones se encuentran, como autora: "Ejes para reconstruir la historia del movimiento de familiares y víctimas de la represión estatal en México", Luchas por la memoria en México, Ciudad de México, El Colegio de México (próximamente); como coautora: "The Liberation of Women: Kollontai in Mexico", en Dora García (ed.), Amor Rojo. Mad Marginal, Berlín, K-Verlag, pp.118-145 (2021); "Las revistas del comunismo", en Carlos Illades (ed.), Camaradas. Nueva historia del comunismo en México, Ciudad de México, Fondo de Cultura Económica pp. 237-265 (2017). 